\title{
An Innovative Framework Supporting SME Networks for Complex Product Manufacturing
}

\author{
Luis Maia Carneiro ${ }^{1}$, Ricardo Almeida ${ }^{1,2}$, Américo Lopes Azevedo ${ }^{1,2}$, \\ Timo Kankaanpaa ${ }^{3}$, and A.H.M. Shamsuzzoha ${ }^{3}$ \\ ${ }^{1}$ INESC Porto, Portugal \\ ${ }^{2}$ FEUP, Portugal \\ ${ }^{3}$ University of Vaasa, Finland \\ luis.carneiro@inescporto.pt, timo.kankaanpaa@uwasa.fi, \\ ahsh@uwasa.fi
}

\begin{abstract}
Current market dynamics require European SME's to focus on complex products manufacturing and to build non-hierarchical business networks, to assure competitiveness and sustainability. Such trend demands appropriate methods for network formation and management, including reference collaboration processes and supporting ICT tools. This paper presents a framework to support SME's in the creation and management of nonhierarchical networks designed and developed within the European RTD project Net-Challenge. This framework includes methodologies, processes and ICT decision support tools aiming at the efficient creation and management of these networks assuring quick response times and competitive and differentiated offerings.
\end{abstract}

Keywords: complex manufacturing, dynamic networks, non-hierarchical business networks, sustainability.

\section{Introduction}

In the context of today's complex product manufacturing, SMEs will have to adopt new business models and to establish dynamic and non-hierarchical networks to respond to market opportunities, assuring quick response, fast time to market, differentiated offerings and competitive prices. Sustainability for SMEs will be found in high-variety low-volume businesses, related with complex products manufacturing [1], [2], [3].

The major problems when dealing with complex products in traditional manufacturing environments are usually the long lead times that require long planning horizons and the multiplicative effects of dependent demands of product components. Small disturbances on higher level components can lead to major problems in the supply network (i.e. the "whip effect" can have even more impact). Furthermore, high variety and low volume requirements can considerably increase the complexity of planning and imply the need for highly flexible resource configurations.

This environment where dynamic networks of SMEs emerge to respond to market opportunities will only work in practice if companies are provided with appropriate methods for network formation and management, reference collaboration processes and 
supporting ICT tools. However, there were no proven and effective methodologies, approaches or tools to support SMEs in creating, managing and dissolving this type of dynamic and non-hierarchical networks for complex products manufacturing.

This study presents a framework being designed and developed by the European RTD project NET-Challenge - Innovative networks of SMEs for complex products manufacturing that aims to fill this gap with the design of effective methodologies, processes and IT decision support tools. It presents an innovative architecture to ensure communication and operational management between companies included on non-hierarchical business networks, heading to achieve the needed flexibility, agility and sustainability.

\section{The Net-Challenge Project}

Temporary networks or Virtual Organizations (VO) are usually set up to dynamically respond to emerging market opportunities. The way companies react to these opportunities is critical to their performance in different phases of the network lifecycle (creating, managing and dissolving).

Net-Challenge is a European research project in the area of collaborative business networks; whose objective is to design, develop, validate and disseminate an integrated framework to support SMEs in creating, managing and dissolving dynamic and non-hierarchical networks for complex products manufacturing. This framework includes the following main components:

- A methodology to help SMEs in partner qualification, formation and operation of dynamic networks (able to quickly respond to market opportunities characterized by low volume, high variety and customer centered products);

- Reference collaboration processes for non-hierarchical networks, to be used to promote and facilitate real collaborative business processes;

- Distributed decision support tools to help companies to manage manufacturing and logistics processes, including: aggregate collaborative planning with dynamic capacity management and real-time order promising; real-time monitoring integrated with event management and performance management.

\section{Business Requirements}

\subsection{Scope}

Business requirements define the high-level needs and features, by focusing on the capabilities needed by the stakeholders and key-users. This chapter aims to define the major needs to promote collaboration in non-hierarchical networks for complex products manufacturing.

To ensure a user driven approach, three Business Cases of non-hierarchical business networks aiming at the design and manufacturing of complex products were defined within the project. The business cases were selected to be representative of a 
large variety of industries and include the following sectors: textile, apparel and wearable technologies (Portugal), customized footwear (Italy) and machine tools (Spain). In each business field it was selected two case companies, acting in the same network. These companies played a key role in requirements analysis and will test and demonstrate the final results. The relations of these companies with their business partners was analyzed in detail, in order to deeply understand the present and potential future business networks these companies can promote [5].

\subsection{Approach to Requirements Analysis}

The approach used for this study (presented on Figure 1) was based, initially, on a research for current trends related with business collaboration and respective support tools, in order to define a target domain.

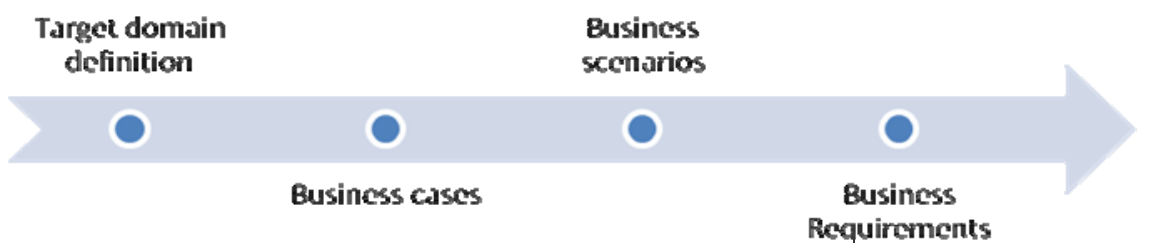

Fig. 1. Approach to requirements analysis

The second step was the detailed analysis of the case companies and the Business cases pilot networks. This was done through detailed interviews with key persons in the case companies and some of their business partners, following structured and detailed interview guidelines. The study was conducted through multiple case study approach, gathering and comparing retrieved information, and sharing the initial findings of the case companies.

The following step included the definition and discussion of future scenarios related with the creation and management of business networks. These future scenarios are like "short stories", easily understood and discussed with companies for a complete validation. Meetings with companies were organized to build and validate the respective scenarios and Skype meetings with RTD partners from the different countries were organized to harmonize and define integrated scenarios.

The analysis of the companies and respective networks and the analysis of the defined future scenarios were done to define the requirements for the Net-Challenge Framework.

\subsection{Business Requirements}

As an output from business scenarios and case company analysis, it was developed a single requirements list, which was submitted to a classification stage. The main objective was to identify the most important requirements for companies. Figure 2 presents a summary of the identified requirements. 


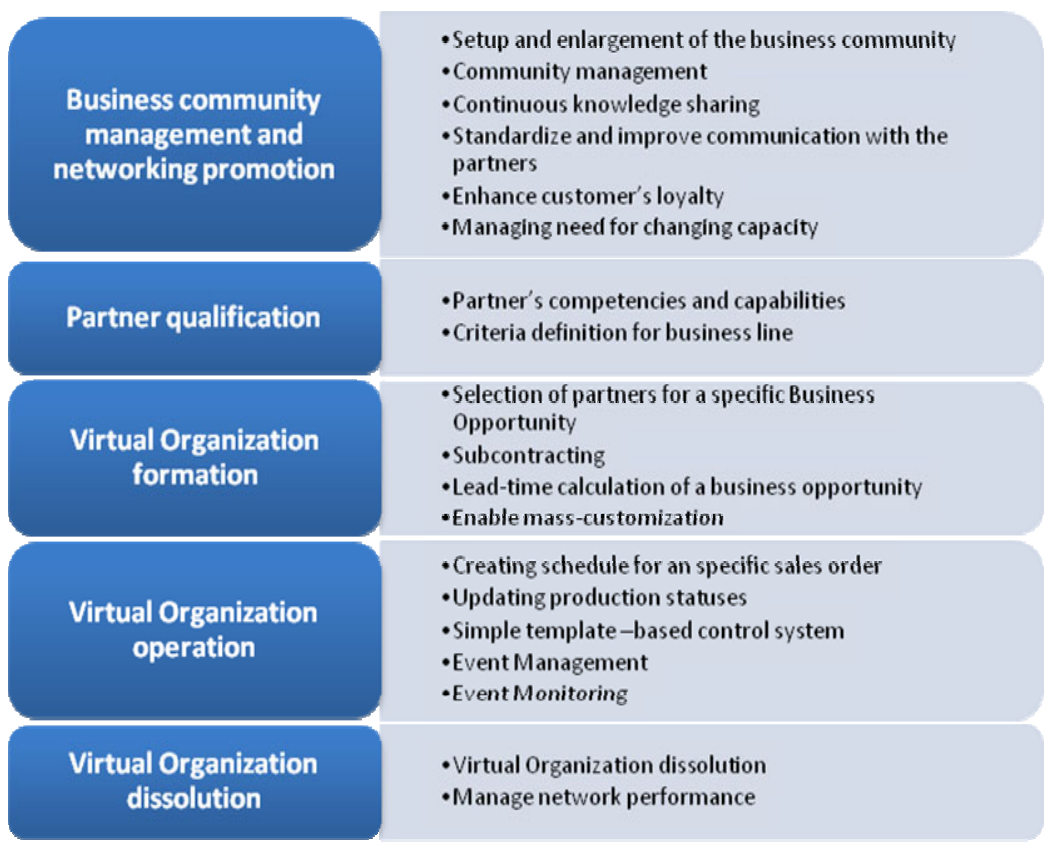

Fig. 2. Business requirements

The requirements were then prioritized with the case companies by clarifying the main focus of the requirement. The case companies gave the highest priority to the following requirements (in the order of importance): 1) Updating production statuses, 2) Selection of partners for a specific business opportunity, 3) Lead-time calculation of a business opportunity, 4) Setup and enlargement of the business community, 5) Standardize and improve communication with the partners.

\section{Proposed Framework}

\subsection{Concept}

Innovative methods and collaborative processes are specifically designed for complex and customized products because they address very important market segments for European SMEs' differentiation and sustainability.

The proposed approach assumes that the relations between participating companies are non-hierarchical and the decision making processes are decentralized. Individual companies must be able to find and receive the commitment of the required partner companies (in terms of competencies and available capacity) to respond to all potentially interesting market opportunities they are aware of in real time. SMEs will only be able to assure differentiation and long term sustainably through the ability to efficiently and effectively form these dynamic networks. 
The vision defines the development of regional or sectorial Business Communities, where trust can be built and communication streamlined, as critical for the fast and efficient creation of these business networks.

The proposed framework is composed of three main components: a Methodology, reference business processes and IT decision support tools.

The proposed approach defines 5 main phases for the successful implementation of non-hierarchical networks of SMEs for high-variety, low-volume and complex products manufacturing, which are identified in the Methodology phases below.

\subsection{The Net-Challenge Methodology}

The proposed Methodology is structured in the main five phases of the Framework which are linked with the lifecycle of these networks.

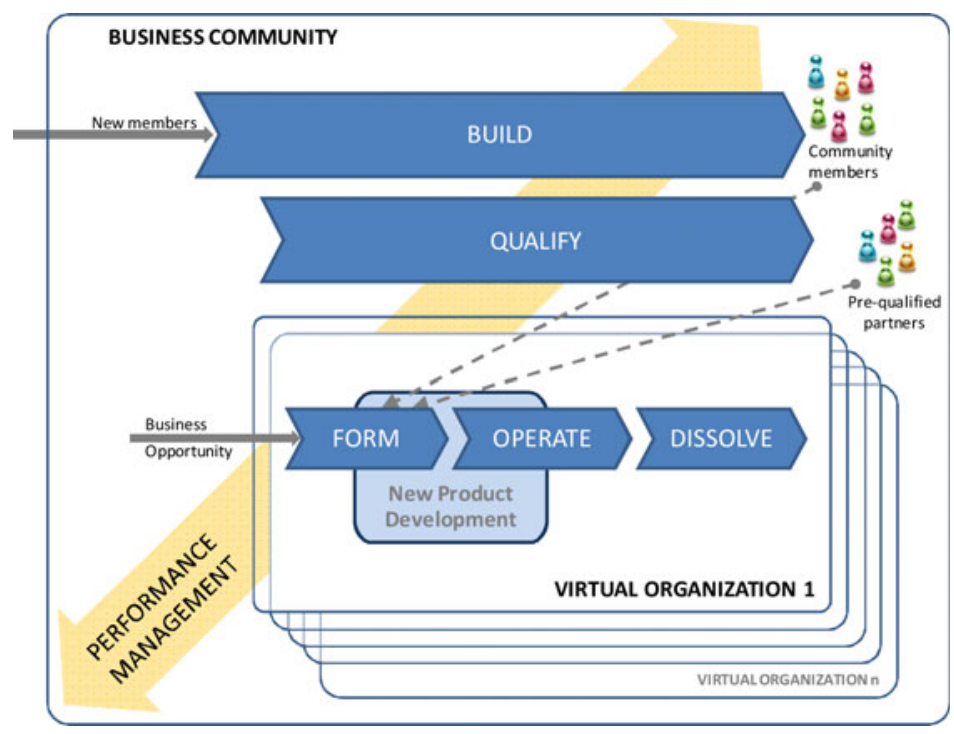

Fig. 3. The Methodology high-level structure

\section{Phase 1: Build and develop a Business Community}

The establishment of dynamic Business Communities is an important pre-requisite for the fast and efficient formation of temporary Virtual Organizations able to successfully respond to specific market opportunities. In this sense, the Building of Business Communities is seen as a crucial enabler for collaboration.

These Business Communities are business environments that comprise a significant number of organizations (mostly SMEs) where trust is developed and communication mechanisms are established. There should be easy mechanisms for finding business partners. This phase specifies the life-cycle of a Business Community and the needed activities and initiatives that need to be implemented to assure its desired performance [4], [6], [7]. Market research activities and sharing of 
business opportunities is promoted. Social networking and knowledge sharing is supported by relevant ICT tools.

\section{Phase 2: Qualify}

During all phases of non-hierarchical networks' lifecycle (and all phases of the Methodology) information about each company participating in the business community is collected and stored. This information will be stored in suitable information systems and a profile of each community member will be created and enriched over time.

For some critical business lines and/or operations community members need to ensure and develop the competencies and capabilities of potential partners. In other words, they need an innovative and collaborative qualification of potential partners. In those cases, companies will only consider qualified partners, instead of working with any member of the Business Community.

\section{Phase 3: Form}

The Form phase is responsible for the creation of a Virtual Organization to respond to a specific market opportunity. In the Form phase a group of partners are interactively selected to collaborate in order to develop and produce a product or collection of products [4], [8]. For complex products the collaborative product design activities assume a very important role. In the innovative approach designed, the product concept is defined in the Form phase to allow the rough plan of the VO and cost estimation. The detail engineering is carried out in the Operate phase.

For complex products manufacturing two complementary scenarios were defined:

(i) One-of-a-kind custom made products that are handled by an ETO (Engineer to Order) strategy.

(ii) Customized products, where a product platform is defined and the requirements from different customers are solved by ATO (Assemble to Order) strategies. The platform enables the customers to specify their needs based on the parameters and options available (customization). The routing and the bill-of-material (BOM) are generated automatically based on the customer selections.

\section{Phase 4: Operate}

The Operate phase is related with the execution of the planned operations to respond to a specific market opportunity.

The approach for Operation is different for the scenarios defined for one-of-a-kind and customized products. Operation starts by the detailed product engineering on the first case and with the engineering of the product platform on the second case.

On the first case, a detailed plan is defined, executed and monitored. On the second case different customers can place orders over time, which are individually planned and executed. In this second case the VO is kept active until there is sufficient demand for the designed product platform. In both cases unforeseen events are monitored and handled.

\section{Phase 5: Dissolve}

Virtual Organizations are created to accomplish a mission and not to operate forever. This mission is linked to responding to a Business Opportunity and should be 
included in the contract signed by partners when forming the VO. Therefore, this contract should also define when the VO will be dissolved as well as the strategies to follow in case other reasons provoke a premature dissolution. In this phase it is of paramount importance the activities related with performance evaluation and sharing of the knowledge accumulated.

\subsection{Reference Collaboration Processes}

Reference collaboration processes are defined for non-hierarchical business networks, promoting and facilitating real collaborative business processes, supporting the high level phases defined in the Methodology.

These reference collaboration processes are designed to allow an easy adaptation to each specific business network. The following areas are covered: Building and managing Business Communities (activities related with creating the business community, membership and profile management, knowledge management); Partner qualification (collaborative assessment and continuous improvement); VO Formation (activities related with product concept definition, technical validation of partner's capabilities and VO's rough plan); Order management and planning processes (activities which include order deployment, collaborative fine planning, monitoring production status); Event management (classification of events, procedures to be performed, handling of unexpected events) and Performance management (aiming at strategic and operational business alignment and continuous improvement).

\subsection{IT Decision Support Tools}

The proposed system architecture includes 4 major areas: Business Community management and integration infrastructure, Aggregate Collaborative Planning (ability of all partners to share and provide a common answer concerning production planning issues), Real Time Monitoring and Event Handling (accurate control of production status and event management, to promote quick answer to unexpected events) and Performance Management (which will constantly update network and partner's performance, for feedback purposes and future analysis, improvement and partner qualification).

For the management of the Business Community, the system concept includes social networking and knowledge management platforms. It supports the management of the community, including membership and profile management.

Taking into account this particular environment, the proposed Decision Support Tools need to be distributed. Each company will be provided with the set of key ICT tools to manage its collaborative activities, to integrate with traditional legacy systems, to interface with business partners and to support decision making. For company a rough capacity model is defined and updated in real time, to enable the real time information on available capacity.

The system architecture is defined to be simple and affordable, and applicable to most European SMEs. Available standards and interoperability frameworks are proposed, such as TexWeave and Shoenet data models, SOA and the ebXML (Electronic Business XML-defined by United Nations and OASIS) framework. 


\section{Conclusions and Future Developments}

Dynamic and non-hierarchical business networks for complex products design and manufacturing is proposed as the key for European SMEs sustainability.

This paper presents the framework being designed and developed by the NetChallenge European project, including effective methodologies, processes and ICT decision support tools, aiming at the successful and efficient implementation of such networks. It includes the development of an innovative architecture to ensure communication and operational management between companies included on nonhierarchical business networks, heading to achieve the needed flexibility and agility to company's sustainability.

This study relies on the conceptual framework of the Net-Challenge project, following the identified specific requirements for complex and customized products.

This project aims to apply their study results on real business scenarios; therefore, it's expected to validate the proposed methodologies, processes and ICT solutions on pilot companies, in order to evaluate an effective application.

\section{Acknowledgements}

The authors would like to acknowledge the co-funding of the European Commission within NMP priority of the Seventh RTD Framework Programme (2007-13) for the Net Challenge project (Innovative Networks of SMEs for Complex Products Manufacturing), Ref. CP-FP 229287-2. The authors also extend their acknowledgments to anonymous reviewers for their helpful comments and support.

\section{References}

1. Gunasekaran, A., Lai, K.-H., Cheng, E.: Responsive supply chain: A competitive strategy in a networked economy. Omega 36(4), 549-564 (2008)

2. Görlitz, O., Neubert, R., Benn, W.: Co-operation Formation in Nonhierarchical Production Networks. In: Proceedings of the eBusiness and eWork Conference (2004)

3. Myers, J.: Future Value Systems: Next Generation Economic Growth Engines \& Manufacturing. In: Proceedings of the IMS Vision Forum 2006, Seoul, Korea, pp. 30-47 (2006)

4. Camarinha-Matos, L.M., Afsarmanesh, H., Ollus, M.: Ecolead and CNO Base Concepts. In: Methods and Tools for Collaborative Networked Organizations. Springer Science+Business Media, LLC 2008, Heidelberg (2008)

5. Carneiro, L., Kankaanpää, T., Almeida, R., Ferreira, P., Loichate, M., Pecina, M., Fornasiero, R., Zaniacomi, A., Chiodi, A., Rymaszewska, A.: Specification of Business Cases. Delivery D1.2. NetChallenge Project (2009)

6. Julka, N., Baines, T., Tjahono, B., et al.: A review of multi-factor capacity expansion models for manufacturing plants. Int. J. Prod. Econ. 106(2), 607-621 (2007)

7. Van Mieghem, J.A.: Capacity management, investment, and hedging: Review and recent developments. Manuf. Serv. Oper. Manag. 5(4), 269-302 (2003)

8. Chen, Q.X., Chen, X., Lee, W.B.: Qualitative search algorithms for partner selection and task allocation in the formulation of virtual enterprise. International Journal of Computer Integrated Manufacturing 2(20) (2007) 